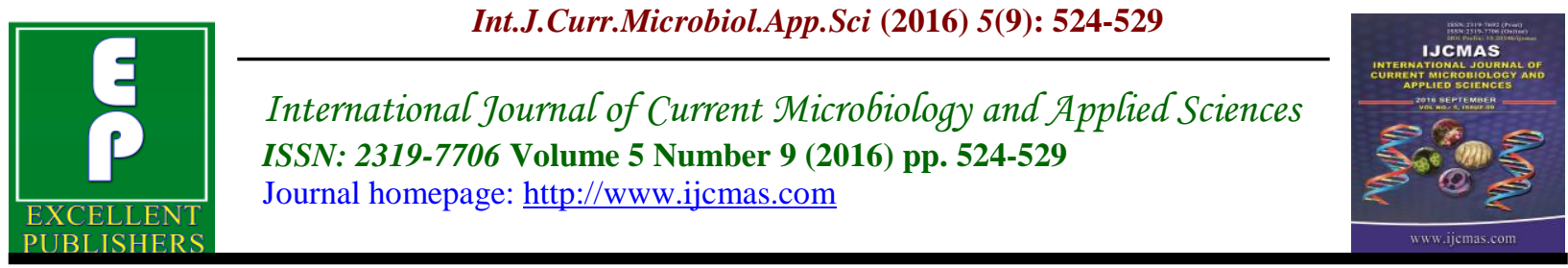

Original Research Article

http://dx.doi.org/10.20546/ijcmas.2016.509.058

\title{
Prevalence of Cryptosporidium spp. among Diarrhea Patients attending Hospital and Community based in Cachar District of Assam, India
}

\author{
Gulzar Hussain $^{1,2}$, Shubhadeep Roychoudhury ${ }^{2}$ and Baby Singha ${ }^{1 *}$ \\ ${ }^{1}$ Molecular Parasitology Laboratory, Department of Zoology, G. C. College, Silchar, India \\ ${ }^{2}$ Department of Life Science and Bioinformatics, Assam University, Silchar, India \\ *Corresponding author
}

Keywords

Cryptosporidium

spp.

Diarrhoea,

Intestinal

parasites.

Article Info

Accepted:

20 August 2016

Available Online:

10 September 2016

\section{A B S T R A C T}

Diagnosis of Cryptosporidiosis and associated diarrhea is a big challenge for health care in this region. To determine the prevalence of cryptosporidiosis among the hospital in the Cachar district of Assam, India. A standard conventional technique was used in the identification of parasites, including cryptosporidiosis from among the 220 diarrheal patients whose samples were analyzed. We report a $65.9 \%$ (145 out of 220) overall parasite, prevalence and $16.3 \%$ (36 out of 220) prevalence of Cryptosporidium oocysts was found in the patients studied. High percentages of Cryptosporidium positive cases were observed in the $<1$ years $(31.3 \%)$ and the 1 14 years $49(22.2 \%)$ age groups. Other intestinal parasites found in the stool samples of the studied diarrhea patients were: hookworm which was observed in 25(11.3\%) patients, Ascaris lumbricoides also in 25(11.3\%) patients, Entamoeba histolytica was found in 19(8.6\%) patients, Giardia lamblia in 11(5.0\%) patients, Cyclospora cayetanensis in $07(3.1 \%)$ patients, Strongyloides stercolaris in 5 (2.2\%), and Trichuris trichiura was found in 09(4.09\%) patients. Poor hygiene both personal and environmental, poor diagnostic, clinical, prevention and control skills facilities may have impacted on Cryptosporidiosis has been reported in this study.

\section{Introduction}

Cryptosporidium spp, an apicomplexan protozoon, parasites causes acute and persistent diarrhoea in the young of several animal species (Angus et al., 1983) and lifethreatening chronic diarrhoea in immunocompromised individuals (Goodgame et al., 1995). The global prevalence of this parasite can be ascertained by the reports from all continents (Current et al., 1991). Intestinal parasitic infections are among the most common infections in the worldwide. It is estimated that 3.5 million peoples in the world are affected as a result of these infections, the majority being children (WHO, 1998). The main clinical sign of these disease caused by the parasites is diarrhea (Al-Shammari et al., 2001). It has effect particularly on infants, and elder people and annually killing at least 4-5 million of those aged less than 5 years in Asia, Africa, and Latin America (Bern et al., 
1992).The main problems in developing countries such as India, particularly in this region where humid condition, the unhygienic condition, and the poor socioeconomic conditions are prone to this parasite. Intestinal parasitic infections cause severe diarrhea, especially in infants, and can be fatal in acute diseases (Adamu et al., 2005).

In this study, we investigate the prevalence of Cryptosporidium spp. with special importance to intestinal parasites in all age group.

\section{Materials and Methods}

A cross-sectional study was conducted from January 2012 to January 2013. A total number of 220 patients visiting pediatric OPD or admitted at pediatric ward, diarrhea ward at Silchar Medical College and Hospital, SMD Civil Hospital, Silchar, Narisikha and various communities in this region of Cachar district Assam, India, having complaints of diarrhea, weight loss, anorexia, and other conditions, which may raise thought of intestinal parasitic infection, were included in this study. The clinical history along with a questionnaire form containing various details of sociodemographic factors were obtained. Stool samples were examined for the presence of Cryptosporidium oocysts and other parasites. Saline and iodine mount preparations were examined microscopically under a $100 \mathrm{x}$ magnification with oil immersion to detect oocysts and/or parasites before and after concentration of the sample of the formal ether technique. The modified Ziehl-Neelsen staining was used for detecting coccidian parasites.

\section{Results and Discussion}

The study was conducted a total number of 220 patients enrolled in the various Hospital and community level of the Cachar districts,
Assam, India, $116(52.72 \%)$ were males and $104(47.27 \%)$ were females (Table 1$)$.

As shown in Table 2, highest number of parasitic infections was seen in age group of, $<1$ years and 1-14 years. This can be characterized to attribute that the school going age and higher outdoor activities the children are more prone to these diseases. In addition, parasitic infections were found to be more in males.

We report a $65.90 \%$ (145 out of 220) overall parasite prevalence and 16.3\% (36 out of 220) prevalence of Cryptosporidium oocysts was found in the patients studied. No parasite was detected in $44(20 \%)$ patients investigated. The age range was between $<1$ years to 84 years. The majority $81(36.8 \%)$ and $72(32.7 \%)$ of the patients were between $<1$ and 25-34 years respectively. The majority of the study population presented with persistent diarrhea, 132 (61.36\%). Other intestinal parasites found to be causing diarrhea alongside Cryptosporidium are hookworm and Ascaris lumbricoides with 25 (11.3\%) prevalence each. Entamoeba histolytica was found in 19 $(8.6 \%)$ patients, Giardia lamblia in $14(6.3 \%)$ patients, Isospora belli in $11(5.0 \%), \quad$ Cyclospora cayetanensis in $8(3.6 \%)$ patients, Strongyloides stercolaris in $3(1.3 \%)$, and Trichuris trichiura was found in $6(2.7 \%)$ patients. (Figure 2 and table 2).

The study also reveals that cryptosporidiosis is higher people of poor socioeconomic status, out of 36 cases of Cryptosporidiosis from the study, 24 (66.6\%) was found to be associated with poor living conditions, they use contaminated drinking water some time direct from the pond, river and marsh which may be contaminated with parasites and these increase the risk of infection. In this study the people of lower educational levels were found to be associated with 
Cryptosporidiosis, out of 36 cases, 27 patients $(75.0 \%)$ was found to be having lower educational background as compared to only 6 patients $(16.6 \%)$.

Cryptosporidium was the most commonly detected diarrhoeagen amongst the various intestinal parasites which were for in the present study. Its role as a diarrhoeagen at high frequency has also been reported from hospitals in northern (Uppal et al., 1991), southern (Mathan et al., 1985), eastern (Das et al., 1993) and western (Sarawathi, 1988) India (range of detection 4.3-13.0\%).

Intestinal protozoan parasites are widespread in the worldwide and remain key role in human health concern in many tropical and subtropical countries. The prevalence rates of intestinal parasitic infections and type of parasite exhibit wide variation from different countries, between geographical areas, communities, and even seasons (Tappe et al., 2011).

In this study, prevalence of intestinal parasites is 65.9\%; among these, $8.6 \%$ are Entamoeba histolytica, 5\% are Giardia lamblia, $16.3 \%$ Cryptosporidium spp., $11.3 \%$ Hookworm $4.09 \%$ Trichuris trichiura $0.36 \%$ Ascaris lumbricoides. $3.1 \%$ Cyclospora cayetanensis, and $2.2 \%$ Strongyloides starcorales.

Table.1 Age and sex distribution in the study population

\begin{tabular}{|c|c|c|c|}
\hline $\begin{array}{c}\text { Age } \\
\text { groups }\end{array}$ & $\begin{array}{c}\text { Percentage }(\%) \\
\mathrm{N}=65.9 \%\end{array}$ & $\begin{array}{c}\text { Male } \\
\mathrm{n}=116\end{array}$ & $\begin{array}{c}\text { Female } \\
\mathrm{n}=104\end{array}$ \\
\hline$<1$ & $69(31.3 \%)$ & $31(14.09 \%)$ & $38(17.2 \%)$ \\
\hline $1-14$ & $49(22.2 \%)$ & $25(11.36 \%)$ & $24(10.9 \%)$ \\
\hline $15-24$ & $34(14.5 \%)$ & $22(10.0 \%)$ & $12(5.4 \%)$ \\
\hline $24-34$ & $23(10.45 \%)$ & $11(5.0 \%)$ & $12(5.4 \%)$ \\
\hline $35-44$ & $19(8.6 \%)$ & $09(4.09 \%)$ & $10(4.54)$ \\
\hline $45-54$ & $13(5.9 \%)$ & $07(3.18 \%)$ & $06(2.7 \%)$ \\
\hline $55-64$ & $08(3.6 \%)$ & $06(2.7 \%)$ & $02(0.90 \%)$ \\
\hline $65-74$ & $04(1.8)$ & $04(1.8 \%)$ & $0(0.0 \%)$ \\
\hline $75-84$ & $01(0.45)$ & $01(0.45)$ & $0(0.0 \%)$ \\
\hline
\end{tabular}

Key; \%=percentage, $\mathrm{n}=$ number

Table.2 Distribution of Cryptosporidium with other intestinal parasites according to sex

\begin{tabular}{|l|c|c|c|}
\hline \multirow{2}{*}{ Parasites } & \multicolumn{2}{|c|}{ Patients } & \multirow{2}{*}{ Total ( $\mathrm{n}=145)$} \\
\cline { 2 - 3 } & Male $(\mathrm{n}=78)$ & Female $(\mathrm{n}=67)$ & \\
\hline Cryptosporidium spp. & $31(14.09 \%)$ & $05(2.27 \%)$ & $36(16.3 \%)$ \\
\hline Hookworm & $04(1.8 \%)$ & $21(9.5 \%)$ & $25(11.3 \%)$ \\
\hline Ascaris lumbricoides & $15(6.8 \%)$ & $10(4.5 \%)$ & $25(11.3 \%)$ \\
\hline Cyclospora cayetanensis & $01(0.45 \%)$ & $06(2.7 \%)$ & $07(3.1 \%)$ \\
\hline Giardia lamblia & $09(4.09 \%)$ & $02(0.09 \%)$ & $11(5.0 \%)$ \\
\hline Strongyloides stercorales & $0(0.0)$ & $05(2.27 \%)$ & $05(2.2 \%)$ \\
\hline Entamoeba histolytica & $17(7.7 \%)$ & $02(0.90 \%)$ & $19(8.6 \%)$ \\
\hline Trichuris trichiura & $0(0.0)$ & $09(4.09 \%)$ & $09(4.09 \%)$ \\
\hline Ascaris lumbricoides & $01(0.45 \%)$ & $07(3.1 \%)$ & $08(0.36 \%)$ \\
\hline
\end{tabular}

Key; \%=percentage, $\mathrm{n}=$ number 
Fig.1 Microscopy (10*100X magnification) using M-zn staining technique. A-B: Cysts of Cryptosporidium spp.

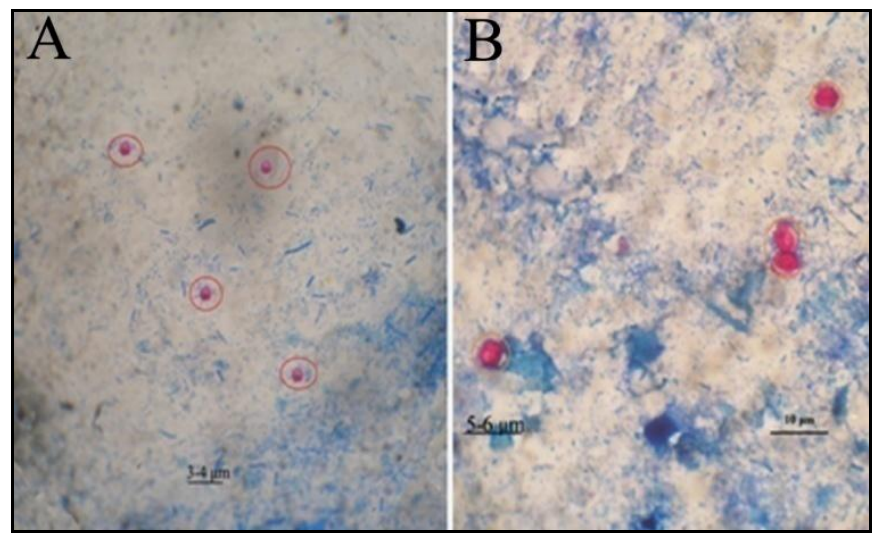

Fig.2 Prevalence of Cryptosporidium spp. and other parasites

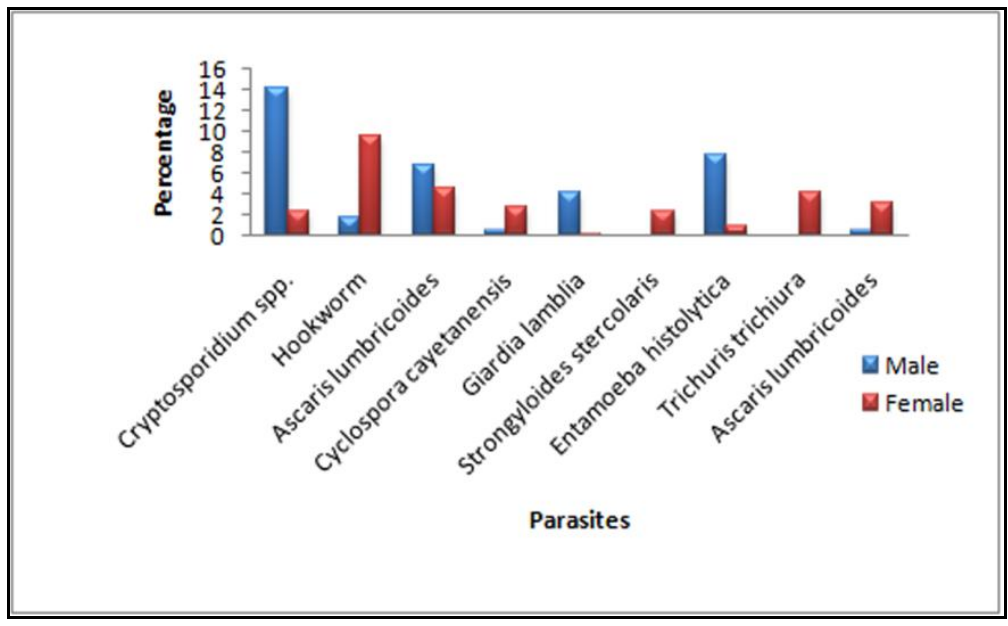

In $40 \%$ of the cases, the nuclear family was found to be uneducated, which leads to poor living conditions and poor sanitary habits, ultimately increasing the risk of parasitic infections. About $54 \%$ cases were living in hurt; such type of hurt tends to be in surrounding area without proper sanitation and drainage system, which contribute to higher prevalence of parasitic infection (Champa et al., 2012 and Kiran et al., 2014). It was also noted that $46.6 \%$ cases where having drinking water from open pond, river and marshes and 20\% were using water from borewell. Because these wells are constructed at a shallow level, they can come in contact with condition for survival and development of eggs like Ascariasis lumbricoides (Awasthi et al., 2008).

In this study, $16.3 \%$ of cryptosporidiosis was reported among the population of mostly affected aged $<1$ years and 1-14 years. This is higher than the report of $1 \%$ from England and 10\% from Peru (Kortbeek et al., 2000 and Moseier et al., 2000). Factors which help in parasite transmission include transmission in day care centers, swimming pools, public water supplies, and other water sources. Cryptosporidium was detected by modified acid fast staining of 
fecal smears in only $2 \%$ of samples analyzed in Accra, Ghana (Binka et al., 2011) compared to $16.3 \%$ obtained in our study with the same detection protocol of modified acid fast staining of fecal smears. This high prevalence may be due to under reporting, poor diagnostic skills caused indirectly by poor resources regarding proper management to control of the disease in developing countries. If use of improved better diagnostic methods such as having high sensitivity must be used to diagnose parasitic infections in patients with diarrhea.

\section{Acknowledgements}

Authors acknowledge the help from the Department of Pediatrics, Microbiology, Silchar Medical College and Hospital in collection of the stool samples.

\section{References}

Adamu, H., Endeshaw, T., Teka, T., Kifle, A., Petros, B. 2005. The prevalence of intestinal parasites in pediatric diarrheal and non-diarrheal patients Addis Ababa Hospitals with special emphasis on opportunistic parasitic infections and with insight into the demographic and socio-economic factors. Ethiap. J. Health Dev., 20(1): 39-46.

Al-Shammari, S., Khoja, T., El-Khwasky, F., Gad, A. 2001. Intestinal parasitic diseases in Riyadh, Saudi Arabia: prevalence, sociodemographic and environmental associates. Trop. Med. Int. Health, 6(3): 184-9.

Angus, K.W. 1983. Cryptosporidiosis in man, domestic animals and birds: a review. J. R. Soc. Med., 76: 62-70.

Awasthi, S., Verma, T., Kotecha, P.V., Venkatesh, V., Joshi, V., Roy, S. 2008. Prevalence and risk factors associated with worm infestation in pre-school children (6-23 months) in selected blocks of Uttar Pradesh and Jharkhand, India. Indian J. Med. Sci., 62(12): 484-90.

Bern, C., Martines, J., de Zoysa, I., Glass, R.I. 1992. The magnitude of the global problem of diarrhoeal disease: a tenyear update. Bull World Health Organ., 70: 705-14.

Binka, E., Vermund, S.H., Armah, G.E. 2011. Rotavirus diarrhea among children less than 5 years of age in urban Ghana. Pediatr. Infect. Dis. J., 30(8): 716-718.

Champa, H., Sreeshma, P. 2012. Intestinal parasitic infections among patients attending a tertiary care hospital in south India. J. Evol. Med. Dental Sci., 1(4): 308-14.

Current, W.L., Garcia, L.S. 1991. Cryptosporidiosis. Clin. Microbiol., Review 4: 325-358.

Das, P., Sengupta, K., Dutta, P., Bhattacharya, M.K., Pal, S.C., Bhattacharya, S.K. 1993. Significance of Cryptosporidium as an aetiologic agent of acute diahorrhoea in Calcutta: a hospital based study. J. Trop. Med. Hyg., 96: 124-127.

Goodgame, R.W., Kimball, K., Ou, C.N. et al. 1995. Intestinal infection and injury in acquired immunodeficiency syndrome-related cryptosporidiosis. Gastroenterol., 108: 1075-1082.

Kiran, T., Shashwati, N., Vishal, B., Ashok kumar, D. 2014. Intestinal parasitic infections and demographic status of school children in Bhopal region of Central India. IOSR J. Pharm. Biol. Sci., 9(5): 83-7.

Kortbeek, L.M. "Clinical representaion in Cryptosporidium-infected patients," in Giardia and Cryptosporidium: From Molecules to Diseases, Ortega-Pierres G, Cacciò SM, Fayer R, Mank TG, Smith HV, and Thompson RCA, Eds., 
chaper XI, pp. 131-137, CABI, Oxfordshire, UK.

Mathan, M.M., Venkatesan, S., George, R., Mathew, M., Mathan, V.I. 1985. Cryptosporidium and diarrhoea in southern Indian children. Lancet, 2: 1172-1175.

Mosier, D.A. and Oberst, R.D. 2000. "Cryptosporidiosis: a global challenge," Annals of the New York Academy of Sciences, vol. 916, pp. 102-111, View at Google Scholar and Scopus.

Sarawathi, K., Pandit, P.V., Deodhar, L.P., Bichile, L.S. 1988. Prevalence of cryptosporidia in patients in Bombay.

Indian J. Med. Res., 87: 221-224. Tappe, K.H., Mohammadzadeh, H., Khashaveh, S., Rezapour, B., Barazesh, A. 2011. Prevalence of intestinal parasitic infection among primary school attending students in Barandoozchay region of Urmia, Weat Azerbaijan province, Iran in 2008. Afr. J. Microbiol. Res., 5(7): 788-91.

Uppal, B., Natrajan, R. 1991. Detection of Cryptosporidium oocyst in acute diarrheal stools. Indian Paediatr., 28: 917-920.

WHO. 1998. Control of Tropical Diseases. Geneva: World Health Organization.

\section{How to cite this article:}

Gulzar Hussain, Shubhadeep Roychoudhury and Baby Singha. 2016. Prevalence of Cryptosporidium spp. among Diarrhea Patients attending Hospital and Community based in Cachar District of Assam, India. Int.J.Curr.Microbiol.App.Sci. 5(9): 524-529. doi: http://dx.doi.org/10.20546/ijcmas.2016.509.058 